\title{
Youth Sport Values Questionnaire-2 (YSVQ-2): evidências de validade para o contexto brasileiro
}

\author{
Marina Pereira Gonçalves \\ Jair Nunes Rocha \\ Paulo Gregório Nascimento da Silva \\ Letícia Coelho de Oliveira \\ Universidade Federal do Vale do São Francisco, PE, Brasil \\ Valeschka Martins Guerra \\ Universidade Federal do Vale do Espírito Santo, ES, Brasil
}

\begin{abstract}
Resumo
Objetivou-se adaptar e validar para o contexto brasileiro o Youth Sport Values Questionnaire-2 (YSVQ-2) por meio de dois estudos: No Estudo 1, contando com a participação de 181 jovens atletas, foi realizada uma Análise de Componentes Principais (ACP) e identificou-se três fatores condizentes com o estudo original. Entretanto, os índices de consistência interna (alpha de Cronbach) foram aceitáveis somente para dois fatores (status: $\alpha=0,62$; moral: $\alpha=0,70$ ), no fator competência este índice foi um pouco abaixo do aceitável $\alpha=0,56$ ). No Estudo 2, buscou-se comprovar a estrutura fatorial do YSVQ-2, contando com uma nova amostra de 200 jovens atletas. Foi realizada uma Análise Fatorial Confirmatória (AFC), onde os resultados demonstraram indicadores de ajuste do modelo próximo ao recomendado: $\chi^{2}(148)=108,43, \mathrm{p}<0,001, \chi^{2} / \mathrm{gl}=2,26$, GFI $=0,92, \mathrm{CFI}=0,90, \mathrm{RMSEA}=0,08$ $(\mathrm{IC} 90 \%=0,06-0,10)$. Assim, o estudo apresentou evidências de validade fatorial do YSVQ-2 para o contexto brasileiro, porém novas pesquisas de validade deste instrumento são recomendadas.
\end{abstract}

Palavras-chave: Análise fatorial; Escalas; Valores; Esportes; Atletas.

\section{Youth Sport Values Questionnaire-2 (YSVQ-2): evidence of validity to a Brazilian context}

\begin{abstract}
The aim of this study was to adapt and to validate the Youth Sport Values Questionnaire-2 (YSVQ-2) in the Brazilian context through two studies: In Study 1, with the participation of 181 young athletes, a Principal Component Analysis (PCA) was performed and three factors were identified that were consistent with the original study. However with internal consistency index (Cronbach's alpha) acceptable only two factors (status: $\alpha=0.62$; moral: $\alpha=0.70$ ), the competence factor was slightly below the acceptable $(\alpha=0.56)$. Study 2 aimed at confirming the factor structure of the YSVQ-2, with a new sample of 200 young athletes. A Confirmatory Factor Analysis was conducted (CFA) and results indicated goodness-of-fit indexes close to the recommended values: $\chi^{2}(148)=108.43, \mathrm{p}<0.001, \chi^{2} / \mathrm{df}=2.26$, GFI $=0.92, \mathrm{CFI}=0.90, \mathrm{RMSEA}=0.08(\mathrm{IC} 90 \%=0.06-0.10)$. Therefore, the study showed evidences of construct validity of the YSVQ-2 to a Brazilian context, however new validity studies of this instrument are recommended.
\end{abstract}

Keywords: Factor analysis; Scales; Values; Sports; Athletes.

\section{Youth Sport Values Questionnaire-2 (Ysvq-2): evidencias de validez para el contexto brasileño}

\section{Resumen}

Se pretendió adaptar y validar para el contexto brasileño el Youth Sport Values Questionnaire-2 (YSVQ-2) por medio de dos estudios: En el Estudio 1, contando con la participación de 181 jóvenes atletas, se realizó un Análisis de Componentes Principales (ACP) y se identificaron tres factores que concuerdan con el estudio original. Sin embargo, los índices de consistencia interna (alfa de Cronbach) fueron aceptables solamente para dos factores (status: $\alpha=0,62$; moral: $\alpha=0,70$ ), en el factor de competencia este índice fue un poco por debajo de lo aceptable $\alpha=0$ 56). En el Estudio 2, se buscó comprobar la estructura factorial del YSVQ-2, contando con una nueva muestra de 200 jóvenes atletas. En el, se realizó un Análisis Factorial Confirmatorio (AFC), donde los resultados mostraron indicadores de ajuste del modelo cerca de la recomendada: $\chi^{2}(148)=108,43, \mathrm{p}<0,001, \chi^{2} / \mathrm{gl}=2,26$, $\mathrm{GFI}=0,92, \mathrm{CFI}=0,90, \mathrm{RMSEA}=0,08(\mathrm{IC} 90 \%=0,06-0,10)$. Así, el estudio presentó evidencias de validez factorial del YSVQ-2 para el contexto brasileño, pero se recomiendan nuevas investigaciones de validez de este instrumento.

Palabras clave: Análisis factorial; Escalas; Valores; Deportes; Atletas. 


\section{Introdução}

Segundo Martin Lee (1996), um dos pioneiros no estudo dos valores no esporte, este é tido como um dos fenômenos sociais mais populares da nossa cultura, se configurando como uma das práticas mais influentes na contemporaneidade. Ao atravessar diferentes grupos e culturas, a prática esportiva tem exercido um papel fundamental na vida das pessoas, sobretudo de indivíduos jovens (Lee, 1996). Dentro desse cenário destaca-se o papel dos valores humanos como elemento norteador para se pensar essa prática, pois ao determinar e justificar as decisões humanas tais valores podem ainda influenciar suas atitudes e comportamentos no meio esportivo (Lee, Whitehead, Ntoumanis, \& Hatzigeorgiadis, 2008).

Como indicado em alguns estudos da área, a atividade esportiva pode vincular-se a alguns processos fundamentais da formação do caráter e do desenvolvimento moral do indivíduo, o que reitera a sua importância enquanto prática que em si mesma tem a capacidade de estimular boas condutas sociais (Lee et al., 2008; Shields \& Bredemeier, 1995). Atrelada a essa concepção positiva relacionada ao esporte ressalta-se a emergência do "fair play", que em linhas gerais se define pelos comportamentos pró-sociais praticados por atletas em contextos competitivos (Kavussanu, Boardley, Sagar, \& Ring, 2013; Lee et al., 2008).

Por outro lado, em detrimento dos "bons" comportamentos, o cenário esportivo, principalmente relacionado aos eventos competitivos, está sendo cada vez mais palco de atitudes negativas no que referem ao fair play, gerando com isso certas condutas consideradas antiéticas. Exemplos desse tipo de postura têm se tornado muito frequentes em escândalos onde atletas utilizam-se de drogas ilícitas para melhorar seu desempenho e performance esportiva (Bardi \& Schwartz, 1994; Dodge \& Robertson, 2004; Hodge \& Lonsdale, 2011; Reardon \& Creado, 2014).

A partir disso, estudos capazes de compreender o papel dos valores dos atletas, tornam-se muito relevantes, uma vez que exploram mecanismos que podem exercer influencias sobre certas condutas ou atitudes em contextos competitivos; e, além disso, o desenvolvimento desse tipo de pesquisa pode auxiliar na produção e aperfeiçoamento de estratégias mais contundentes no que se refere ao aumento de atitudes de fair play entre jovens atletas competidores.

Neste sentido, torna-se importante apontar como os valores têm sido mensurados no contexto esportivo.

\section{Mensuração dos Valores no Esporte}

Ainda é escasso no contexto da literatura científica da área, a utilização e desenvolvimento de instrumentos de medida adequados ao cenário esportivo, isso principalmente no que concerne às investigações sobre os valores no esporte (Lee, Whitehead, \& Balchin, 2013). Apesar dos valores serem considerados um construto fundamental na Psicologia social e apresentar importância no processo de orientação das práticas cotidianas, o mesmo ainda é um conceito que causa equívocos entre psicólogos, principalmente no contexto esportivo, sobretudo, por se aproximar do conceito de atitudes (Lee, Whitehead, \& Balchin, 2013; Gouveia, 2013; Saldanha, Balbinotti, \& Balbinotti, 2015).

Lee et al. (2013), verificaram, por exemplo, que Webb (1969) criou a Profissionalism of Attitudes Scale, com a finalidade de medir atitudes profissionais no esporte. Porém, Lee (1996) percebeu que esta seria essencialmente uma escala de valores, uma vez que o atleta precisaria elencar/escolher o que ele prioriza no esporte: "jogar bem"; "vencer" ou "praticar fair play", indicando ainda certa confusão na compreensão dos valores e das atitudes no esporte.

A este respeito, Gouveia (2013) aponta que as atitudes são provavelmente o construto que mais se confunde com os valores. Entretanto, o que os diferencia é, sobretudo, que as atitudes constituem uma organização de várias crenças em relação a um objeto, uma ideia ou uma situação específica, enquanto os valores transcendem tais elementos específicos, constituindo-se como critérios gerais de orientação (Gouveia, 1998), além de terem um papel central na estrutura cognitiva das pessoas (Rokeach, 1973). Ademais, Gouveia (2013) indica que os valores precedem as atitudes, ou seja, dependendo da prioridade valorativa do indivíduo, este pode ter atitudes diferenciadas frente a algum objeto. Fazendo uma analogia com o contexto esportivo, podemos ter atletas que priorizam valores de justiça, e por isso, podem ter uma atitude favorável à vitória justa, por exemplo.

Assim, no contexto esportivo, na tentativa de desenvolver uma medida de valores no esporte, Simmons e Dickinson (1986, citados por Lee et al., 2013) elaboraram 14 itens com base na teoria de valores de Rockeach (1973). Análises demonstraram que estes são organizados em cinco fatores, porém os mesmos não indicavam que haviam sido elaborados por qualquer base teórica ou a partir de uma população de interesse. Posteriormente, com o intuito de superar as críticas de que tal medida não era representativa da população-alvo, ou seja, dos atletas, Lee e 
Cockmam (1995) com base em Braithwaite e Laws (1985) utilizaram o método de Kolberg para discutir dilemas morais específicos do esporte. Para tanto, realizaram entrevistas semiestruturadas com 87 atletas e identificaram 18 valores, concluindo que um conjunto de valores seria relevante para a tomada de decisões morais no esporte.

Assim, levando em consideração esses estudos e instrumentos anteriores sobre valores no esporte, Lee, Whitehead e Balchin (2000) desenvolveram o Youth Sport Values Questionnaire (YSVQ) com a finalidade de avaliar o sistema de valores de jovens atletas. Esta medida foi posteriormente revisada por Lee et al. (2008) e passou a ser conhecido como Youth Sport Values Questionnaire-2 (YSVQ-2). O desenvolvimento deste instrumento, bem como os seus pressupostos teóricos serão apresentados a seguir.

\section{Youth Sport Values Questionnaire-2 (YSVQ-2)}

Segundo Lee et al., (2013), as estratégias de pesquisa utilizadas para o desenvolvimento desta medida foram derivadas de Rokeach (1973), o qual indica que o princípio base para mensurar a importância relativa dos valores como padrões que guiam os comportamentos das pessoas seria por meio da descrição de um sistema hierárquico de crenças. Esses autores também levaram em consideração o pressuposto de Braithwaite e Law (1985) de que uma medida de valores deve ser derivada da sua população de interesse. Finalmente, com base em Schwartz (2001), os autores consideraram que o formato da escala de resposta pudesse ter um nível de oposição (por exemplo: $\mathbf{- 1}=O$ oposto do que acredito), por ser mais adequado à população jovem e apresentar vantagem para algumas análises estatísticas.

Além disto, na construção da YSVQ original foram realizados estudos qualitativos e quantitativos para se construir os itens e chegar a uma versão capaz de mensurar os valores de jovens no esporte. Entretanto, neste primeiro instrumento havia apenas um item para cada valor, então os autores consideraram que este nova versão (YSVQ-2) deveria representar as três dimensões identificadas em estudos anteriores: valores de moral, competência e status (Lee et al., 2000; Lee et al., 2008).

Posteriormente, Whitehead e Gonçalves (2013) concluíram que as escalas YSVQ e YSVQ-2 representam pontos de referência fundamentais na estrutura dos valores humanos proposta por Rokeach (1973) e estendida por Schwartz (1992). Estes autores argumentam isto a partir de cinco aspectos: 1) os valores de moral e de competência representam os valores interpessoais e intrapessoais de Rokeach, uma vez que os itens de moral das medidas são sociomorais e os itens de competência são autoreferenciados; 2) os valores morais e de status ilustram os polos de oposição autotranscendência e autopromoção da teoria de Schwartz; 3) como o esporte representa uma situação clássica de realização pessoal (McClelland et al., 1953, citados por Whitehead e Gonçalves, 2013), os valores de competência e status representam interpretações intrapessoais e interpessoais de realização. E, apesar de terem sido derivados de dimensões anteriores sobre valores, eles têm alguma correspondência com as metas de orientações à tarefa e ao ego, as quais são consideradas um paradigma central para os estudos a respeito dos valores de jovens atletas por algumas décadas. E, finalmente, 4) os valores da YSVQ-2 representam as três categorias parcimoniosas de Webb (1969) verificadas em um primeiro estudo sobre valores no esporte: jogar com justiça, jogar bem e vencer (Whitehead \& Gonçalves, 2013).

Assim, o YSVQ-2 foi construído a partir desses princípios e baseados nos valores identificados por Lee e Cockman (1995). Esta versão recente é composta por 13 itens, agrupados em três fatores (valores morais, valores de competência e valores de status) e respondidos em uma escala do tipo Likert de 7 pontos (5=Extremamente importante para mim à $\mathbf{- 1}=O$ oposto do que acredito). Este instrumento foi validado em alguns países (Chan, Whitehead, Hatzigeorgiadis, \& Chow, 2013; Gonçalves et al., 2005; Lee et al., 2008; Šukys, 2010) tendo sido verificada a mesma estrutura composta por três fatores (valores de moral, competência e status) e índices psicométricos satisfatórios.

No contexto brasileiro, há apenas um estudo de validação da YSVQ-2 desenvolvido por Saldanha, Balbinotti e Balbinotti (2015). Estes autores realizaram uma tradução e a validade de conteúdo desta escala, a qual reuniu evidências quanto à clareza e pertinência dos itens para medir valores no esporte após uma avaliação feita por juízes (experts) da área. No entanto, não são verificadas informações quanto à comprovação da estrutura fatorial desta medida no contex to brasileiro, o que justifica a realização do presente estudo.

Com o surgimento do YSVQ e YSVQ-2 construídos por Lee e seus colaboradores entre os anos de 2000 e 2008, algumas pesquisas na área de avaliação de valores no esporte puderam ser desenvolvidas, muitas delas apresentando resultados bastante promissores, sobretudo, na compreensão das diferenças dos valores dos atletas segundo o gênero (Pitts, 2015; Suksy \& Jansoniené, 2012) e a idade (MacLean \& Hamm, 
2008; Nyaga, 2011). Bem como, verificando o papel dos valores como preditores de alguns construtos importantes no cenário esportivo, como atitudes morais (Saldanha, 2012; Gonçalves, Oliveira, Ribeiro, Barbosa, \& Silva, 2013), comportamentos pró-sociais e antissociais no esporte (Boardley \& Kavussanu, 2010) e orientação à realização (Whitehead, Lee, \& Hatzigeorgiadis, 2003; Whiteahead, 2016).

Diante do exposto, percebe-se que os esforços de Lee e seus colaboradores para a construção de um questionário sobre valores no esporte têm contribuído para o desenvolvimento dessa área. No entanto, pesquisas sobre valores no esporte ainda são escassas, sobretudo no contexto brasileiro. Neste sentido, justifica-se a realização da presente pesquisa, que tem por objetivo adaptar e apresentar evidência de validade fatorial do Youth Sport Values Questionnaire 2 (YSVQ-2) para o presente contexto, de modo que este possa ser utilizado em futuros estudos visando compreender os valores dos atletas brasileiros.

\section{Método}

Para responder os objetivos desta pesquisa, foram realizados dois estudos empíricos, descritos a seguir.

\section{ESTUDO 1 - Tradução, adaptação e validade fatorial do YSVQ-2}

Inicialmente, foram realizadas as traduções das escalas do inglês para o português por três pesquisadores com conhecimento avançado em língua inglesa, além de uma pessoa cujo idioma nativo é o inglês. Ressalta-se que para as traduções levaram-se em consideração aspectos culturais, ou seja, os itens foram traduzidos sem perder seu sentido original, não sendo feita uma tradução ipsis litteris, tendo o cuidado de utilizar apenas palavras ou expressões comuns à cultura brasileira.

Em seguida, foi realizada uma análise semântica do instrumento, cujo objetivo é verificar se os itens são compreensíveis aos membros da qual se destinará a utilização da medida (Pasquali, 2010). Para tanto, foi solicitado a 10 jovens com idade de 11 onze anos; a 05 adultos praticantes de esportes e a 05 adultos com nível superior de escolaridade, todos foram solicitados a responder o instrumento e apontar o que não estavam compreendendo, e sugerissem possíveis modificações. Após a avaliação desses participantes, foram realizadas pequenas alterações no instrumento, por exemplo, onde aparecia o nome 'jogador' foi trocado por 'atleta' (Eu pretendo me tornar o melhor atleta). Em seguida, com o instrumento piloto pronto, deu-se início a etapa de verificação empírica da escala.

\section{Amostra}

Contou-se com a participação de 181 jovens atletas de diversas modalidades esportivas (individuais e coletivas), com experiência em competições, sendo $63 \%$ do sexo feminino e $37 \%$ do sexo masculino, com idades variando entre 12 e 26 anos $(M=16,54$ anos; $D P=3,53)$. A maioria $(58,3 \%)$ encontrava-se cursando o Ensino Médio e praticava esporte há 1 ano (12 meses). As modalidades esportivas relatadas nos questionários foram: vôlei $(44,2 \%)$, handebol $(21,5 \%)$, futsal $(13,3 \%)$, basquete $(7,2 \%)$, atletismo $(7,7 \%)$, futebol $(4,5 \%)$, judô $(0,6 \%)$ e natação $(0,6 \%)$. Tratou-se, portanto, de uma amostra por conveniência (não-probabilística), sendo incluídos apenas atletas que praticassem esporte com finalidade competitiva, com idade superior a 12 anos e inferior a 26 anos, devido ao instrumento proposto ser apropriado para jovens atletas.

\section{Instrumentos}

Os participantes responderam a versão traduzida e adaptada para a língua portuguesa do Youth Sport Values Questionnaire 2 (YSVQ-2), o mesmo tratase de uma medida que avalia o sistema de valores de jovens atletas, foi desenvolvida por Lee et al. (2008), sendo uma revisão da escala anterior (YSVQ) construída por Lee, Whitehead e Balchin (2000). Esta versão atual é composta por 13 itens, agrupados em três fatores (valores morais $\alpha=0,79$, valores de competência $\alpha=0,74$ e valores de status $\alpha=0,82$ ), respondidos em uma escala do tipo Likert de 7 pontos que varia de $\mathbf{5}=$ Extremamente importante para mim à $\mathbf{- 1}=O$ oposto do que acredito. Tendo apresentado bons indicadores de ajuste ao modelo com esses três fatores: $\left(\chi^{2}=129.43, d f=62, p<0.001\right.$, RMSEA $=0.05$, $\mathrm{SRMR}=0.04, \mathrm{CFI}=0.97, \mathrm{NNFI}=0.96$ ).

Questões Sociodemográficas. Foram solicitadas informações de caráter sociodemográficas (por exemplo, idade, sexo, tipo de modalidade esportiva, experiência no esporte, títulos esportivos, entre outras) com a finalidade de caracterizar a amostra.

\section{Procedimentos}

Para a coleta de dados foi obedecido um procedimento padrão de aplicação, no que diz respeito às medidas utilizadas. Inicialmente, entrou-se em contato com os treinadores e/ou a escola em que o atleta praticava esporte, para saber a disponibilidade dos atletas em responder a pesquisa e ainda se a instituição iria autorizar a aplicação dos questionários em seu estabelecimento. Em seguida, pesquisadores previamente treinados realizaram agendamentos e, 
nas datas marcadas, se dirigiram até o ambiente de treino dos atletas (escola, clube ou outra instituição esportiva) para que estes respondessem os questionários, mediante permissão e assinatura do Termo de Consentimento Livre e Esclarecido pelos maiores de 18 anos e/ou escola ou treinador responsável pelos mesmos. Ressalta-se que esta pesquisa foi aprovada por um Comitê de Ética local, sob o número $0018 / 240811$.

\section{Análise dos dados}

Para verificar a validade fatorial da YSVQ-2 e a sua fidedignidade, foi realizada uma Análise de Componentes Principais, bem como análises dos índices de consistência interna (Alfa de Cronbach) da medida, através do SPSS (Versão 20).

\section{Resultados do ESTUDO 1}

Primeiramente, foram verificados os índices de adequabilidade da amostra, o Kaiser-Meyer-Olkin (KMO) e o Teste de Esfericidade de Bartlett (Pasquali, 2012). Estes indicaram ser possível proceder com a análise fatorial, o $K M O$ foi de 0,71 , verificando-se um qui-quadrado significativo: $\left[\chi^{2}(78)=311,759\right.$; $p<0,001]$. Assim, foi realizada uma Análise de Componentes Principais (ACP) fixada em três fatores com rotação Varimax. Foram considerados itens com cargas fatoriais acima de 0,30 em um único fator
(Pasquali 2010, 2012). De acordo com o critério de Kaiser (valor próprio), foram identificados três componentes explicando $47 \%$ da variância total, bem como segundo o critério de Cattell (distribuição gráfica dos valores próprios) também foram observados três fatores. Porém, devido à fragilidade desses critérios, efetuou-se uma Análise Paralela, considerando 1.000 bancos de dados que simularam a presente pesquisa, ou seja, 181 participantes e 13 itens. Assim, foram gerados sete valores próprios aleatoriamente: 1,58, 1,43, 1,32, $1,23,1,16,1,09$ e 1,03. Contrastando esses valores com aqueles observados empiricamente (Tabela 1), confirma-se a retenção de três componentes, os quais apresentaram valores próprios superiores aos simulados. Na Tabela 1 são apresentados os resultados da ACP e o índice de consistência interna (Alfa de Cronbach) da YSVQ-2.

Ao analisar a Tabela 1, percebe-se que a estrutura composta por três fatores se mostrou adequada para o presente contexto. Dos treze itens que compõem a escala, apenas um deles não se mostrou adequado, a saber, o item 04, uma vez que apresentou carga fatorial abaixo de 0,30 . Os três fatores resultantes da análise fatorial podem ser descritos como segue:

Fator I-Moral: este foi formado pelos itens 07, 02, 12 e 13 (quatro itens), representando a ideia de uma valorização dos aspectos morais que envolvem um jogo competitivo, sua consistência interna (Alpha de Cronbach) foi 0,70 .

TABELA 1

Estrutura fatorial do Youth Sport Values Questionnaire-2.

\begin{tabular}{|c|c|c|c|c|c|}
\hline Itens & Descrição & Moral & Status & Competência & $h^{2}$ \\
\hline YSVQ07 & Eu demostre bom espírito esportivo (jogo limpo). & 0,77 & $-0,01$ & 0,00 & 0,60 \\
\hline YSVQ02 & Eu tente ser justo. & $\mathbf{0 , 7 5}$ & $-0,01$ & 0,18 & 0,60 \\
\hline YSVQ12 & Eu ajude as pessoas quando precisam. & 0,71 & $-0,02$ & 0,10 & 0,52 \\
\hline YSVQ13 & Eu defina minhas próprias metas. & $\mathbf{0 , 5 1}$ & 0,25 & 0,23 & 0,38 \\
\hline YSVQ09 & Eu me veja como sendo o melhor. & $-0,02$ & $\mathbf{0 , 8 3}$ & $-0,06$ & 0,69 \\
\hline YSVQ08 & Eu pretenda me tornar o melhor atleta. & 0,09 & 0,63 & 0,28 & 0,63 \\
\hline YSVQ01 & Eu demonstre que sou melhor do que outros. & 0,09 & 0,60 & $-0,34$ & 0,48 \\
\hline YSVQ11 & Eu ganhe ou venço os outros. & 0,03 & $\mathbf{0 , 5 9}$ & 0,30 & 0,44 \\
\hline YSVQ05 & Eu seja um líder no grupo. & 0,04 & 0,40 & 0,37 & 0,30 \\
\hline YSVQ03 & Eu procure melhorar meu desempenho. & 0,20 & $-0,16$ & 0,78 & 0,67 \\
\hline YSVQ06 & Eu use minhas habilidades bem. & 0,07 & 0,24 & 0,68 & 0,53 \\
\hline YSVQ10 & Eu sempre possa competir corretamente. & 0,32 & 0,08 & 0,45 & 0,31 \\
\hline YSVQ04 & Eu faça o que me mandam fazer. & 0,24 & 0,09 & 0,24 & 0,12 \\
\hline Valor próprio & & 2,15 & 2,12 & 1,84 & \\
\hline V. explicada & & $16,57 \%$ & $16,28 \%$ & $14,14 \%$ & \\
\hline Alfa de Cronbach & & $\alpha=0,70$ & $\alpha=0,63$ & $\alpha=0,56$ & \\
\hline
\end{tabular}


Fator II - Status: este ficou composto pelos itens 05, 09, 08, 01 e 11 (cinco itens), através de suas cargas fatoriais emerge a ideia de valorizar o status no esporte, priorizando a busca unicamente pela vitória e reconhecimento. Sua consistência interna (Alpha de Cronbach) foi 0,63.

Fator III - Competência: e, por último, este fator foi formado pelos itens 03, 06 e 10 (três itens), indicando a priorização de valores relacionados a busca por maior competência no esporte. Sua consistência interna (Alpha de Cronbach) foi 0,57.

A fim de verificar a comprovação da estrutura fatorial da YSVQ-2 para o presente contexto, foi realizado um outro estudo considerando uma nova amostra, este é descrito a seguir.

\section{ESTUDO 2 - Comprovação da Estrutura Fatorial do YSVQ-2}

\section{Amostra}

Participaram deste segundo estudo 200 jovens atletas de diferentes modalidades esportivas (individuais e coletivas), respondendo a versão final do instrumento desenvolvido nas primeiras etapas desta pesquisa. Deste total, $75 \%$ do sexo masculino e $25 \%$ do sexo feminino, com idade média de 24,14 anos $(D P=8,89)$. Em relação à escolaridade, a maioria $(46,0 \%)$ está cursando o Ensino Médio. As modalidades esportivas relatadas nos questionários foram principalmente: Futebol $(31,5 \%)$, atletismo $(17,5 \%)$, vôlei $(9,5 \%)$, artes marciais $(8,5 \%)$, entre outros. O tempo de participação em competições variou de 1 a 42 anos $(m=7,67$ e $d p=7,28)$, sendo estas de diferentes níveis: escolares (15\%), municipais $(17 \%)$, regionais $(17,5 \%)$, estaduais $(31,5)$, nacionais $(18 \%)$, internacionais $(1 \%)$. O critério para seleção da amostra foi o de conveniência (nãoprobabilística), sendo os atletas recrutados a partir da sua concordância e disponibilidade em participar da pesquisa, sendo incluídos apenas aqueles que praticassem esporte com finalidade competitiva e com idade superior a 12 anos, independente de serem amadores ou profissionais.

\section{Instrumentos}

Youth Sport Values Questionnaire - 2 (Questionário de Valores para Jovens no Esporte - 2): versão traduzida e adaptada para o presente contexto, após a realização da análise de componentes principais descrita no ESTUDO 1, onde foram considerados 12 itens (excluindo o item quatro que não se mostrou adequado para o presente contexto) que avaliam valores no esporte a partir de três dimensões valorativas (Valores Morais $\alpha=70$, de Competência $\alpha=57$ e de Status $\alpha=62$ ). Sendo ainda necessário, verificar se o modelo com esses três fatores se adequa aos dados do contexto brasileiro.

Questões sociodemográficas: foi utilizado o mesmo questionário com perguntas de caráter sociodemográfico, descrito no ESTUDO 1.

\section{Procedimentos}

Os dados foram coletados pelos membros do grupo de pesquisa, nos próprios locais de treinos e eventos esportivos, mediante um contato prévio feito com as instituições e equipe técnica responsável. A aplicação seguiu um procedimento padrão que leva em consideração todos os aspectos éticos em pesquisa com humanos. Os atletas foram informados sobre o objetivo geral da pesquisa, bem como fora salientado o caráter confidencial e anônimo dos dados obtidos neste estudo. Diante desses esclarecimentos, os sujeitos assentiram a sua participação na pesquisa a partir da assinatura do Termo de Consentimento Livre e Esclarecido (TCLE) pelos maiores de 18 anos e/ou escola ou treinador responsável pelos mesmos, bem como a anuência dos menores participantes. Ressalta-se que esta pesquisa foi aprovada por comitê de ética local sob o número 0020/121211 CEEHA/UNIVASF.

\section{Análise dos dados}

Foram utilizados os seguintes programas estatísticos para realizar as análises dos dados: SPSS $(P A S W)$ e AMOS (Analysis of Moment Structures) ambos em suas versões 22. Com o PASW foi possível calcular estatísticas descritivas (distribuição de frequência, médias, desvios padrões). Com o AMOS foi realizada a Análise Fatorial Confirmatória (AFC). Neste caso, considerou-se a matriz de variância-covariância como entrada, sendo empregado o estimador ML (Maximum Likelihood). Ademais foram considerados os principais indicadores de ajuste do modelo (Byrne, 2016): (1) $\chi^{2}$ (qui-quadrado), onde o maior valor é indicativo de um modelo insatisfatório; 2 ) $\chi^{2} / \mathrm{gl}$ (graus de liberdade) que é considerado uma bondade de ajuste subjetiva, sendo aceitos índices iguais ou inferiores a 5,$00 ; 3)$ Índice de Bondade de Ajuste (Goodnessof-Fit Index, GFI) e o Índice de Bondade de Ajuste Ponderado (Adjusted Goodness-of-Fit Index, AGFI), que são medidas de variabilidade explicada pelo modelo. Esses índices variam de 0 a 1,00, sendo que são desejáveis valores iguais ou superiores a 0,90 , ou seja, quanto mais próximo de 1,00 os índices são considerados satisfatórios, indicando que o modelo se ajusta aos dados; 4) RMSEA (Root Mean Square Error Approximation) que é baseado na análise dos residuais, quanto menor o seu valor, melhor ajuste 
do modelo, aceitando-se um valor de até 0,10 como adequação do modelo, sendo que abaixo de 0,05 o ajuste é dito ser muito bom. Assim, quanto mais próximo esse índice for de zero, mais o modelo teórico se ajusta aos dados (Hair, Black, Babin, Anderson, \& Tatham, 2009).

\section{Resultados do ESTUDO 2}

Realizou-se uma Análise Fatorial Confirmatória (AFC), considerando o estimador ML (Maximum Likelihood). Os resultados da SEM (Structural Equation Modeling) demonstraram que os indicadores de adequação de ajuste do modelo ficaram próximos ao recomendado: $\chi^{2}(51)=140,01, \mathrm{p}<0,001 ; \chi^{2} / \mathrm{gl}=2,75, \mathrm{GFI}=0,89$, $\mathrm{CFI}=0,86$, RMSEA $=0,09(\mathrm{IC} 90 \%=0,07-0,11)$.

Dessa forma, decidiu-se verificar os Índices de Modificação (IMs) para as saturações (Lambdas, $\lambda$ ) e os erros de medida (Deltas, $\delta$ ) (Hair et al. 2009; Marôco, 2014) a fim de verificar a possibilidade de manter os três fatores propostos no estudo original. Nesse sentido, averiguou-se que seria recomendável realizar três reespecificações no modelo, corre- lacionando, respectivamente, os $\delta$ dos itens 1 e item 7 $(\mathrm{IM}=12,40)$; as saturações dos $\delta$ dos itens 9 e item 11 $(\mathrm{IM}=9,16)$; além dos $\delta$ dos itens 7 e item $12(\mathrm{IM}=8,27)$. Dessa maneira, observou-se uma estrutura fatorial reunindo melhores indicadores de ajuste aos dados empíricos do modelo: $\chi^{2}(148)=108,43, p<0,001$, $\chi^{2} / \mathrm{gl}=2,26, \mathrm{GFI}=0,92, \mathrm{CFI}=0,90, \mathrm{RMSEA}=0,08$ $(\mathrm{IC} 90 \%=0,06-0,10)$. Como é possível comprovar na Figura 1, todas as saturações encontram-se no intervalo comumente esperado (0-1), sendo estatisticamente diferentes de zero $(\lambda \neq 0 ; \mathrm{z}>1,96, p<0,05)$.

Além disto, na Tabela 2 podem ser verificadas informações adicionais sobre os fatores da YSVQ-2.

TABELA 2

Estatísticas descritivas, precisão do construto.

\begin{tabular}{lccccc}
\hline \multicolumn{1}{c}{ Fatores } & $M$ & $D P$ & $\alpha$ & $r_{m . i}$ & $C C$ \\
Moral & 4,35 & 0,47 & 0,73 & 0,42 & 0,75 \\
Status & 2,97 & 1,64 & 0,72 & 0,33 & 0,73 \\
Competência & 4,44 & 4,60 & 0,62 & 0,38 & 0,67 \\
\hline
\end{tabular}

$M=$ média; $D P=$ desvio padrão; $\alpha=$ Alfa de Cronbach $; \mathrm{r}_{\mathrm{m} . \mathrm{i}}=$ índice de homogeneidade; $C C=$ confiabilidade composta.

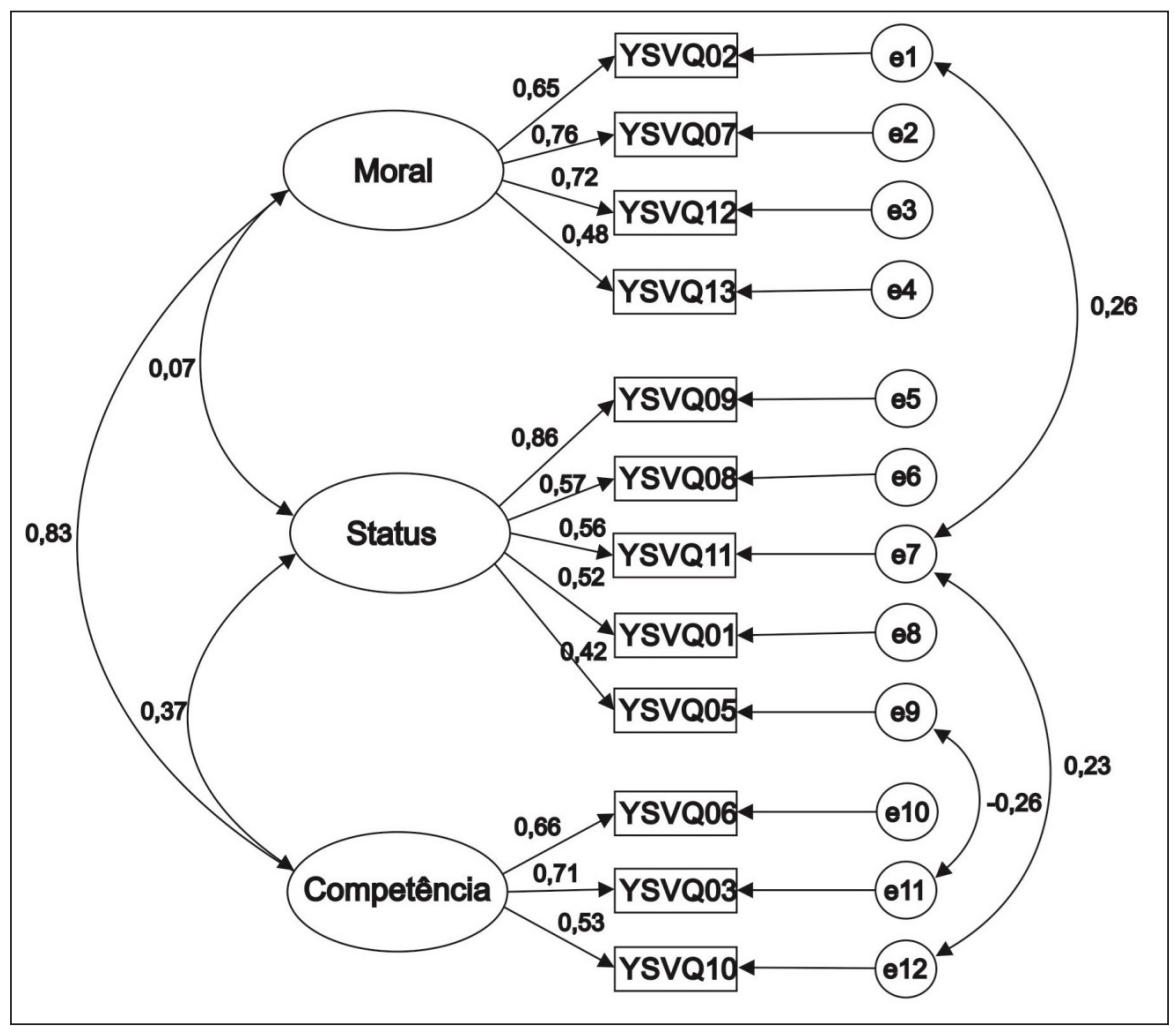

Figura 1. Análise fatorial combinatória do YSVQ-2. 


\section{Discussão e Conclusão}

O objetivo desses dois estudos foi adaptar e validar para o contexto brasileiro o Questionário de Valores para Jovens no Esporte (Youth Sport Values Questionnaire 2-YSVQ-2), buscando verificar evidências de sua validade fatorial e consistência interna. Apesar de tais objetivos terem sido alcançados, é importante ressaltar algumas possíveis limitações. $\mathrm{O}$ fato das amostras terem sido de conveniência (não probabilísticas), onde contamos muitas vezes com a participação de "alunosatletas", ou seja, jovens participantes de competições de nível escolar, não podendo ser considerados atletas jovens de alto rendimento. No entanto, pondera-se que não se pretendia generalizar os resultados, mas avaliar os parâmetros de uma medida específica.

Outra limitação pode ter decorrido dos pressupostos teóricos em que os autores do estudo original utilizaram para construí-la. Segundo Lee, Whitehead e Balchin (2013), as estratégias de pesquisa utilizadas para o desenvolvimento desta medida foram derivadas de Rokeach (1973), Braithwaite e Law (1985) e dos valores identificados por Lee e Cockman (1995), além de considerar o sistema de oposição dos valores de Schwartz (1992). Assim, considera-se que esta mesma medida não foi desenvolvida com base em uma única teoria de valores, o que parece não suportar todas as dimensões do construto valores humanos, uma dificuldade comum para elaborar uma medida psicometricamente adequada (Pasquali, 2010), merecendo atenção no futuro. Neste sentido, esses aspectos podem limitar o poder de generalização dos resultados, porém certamente não os invalidam.

Apesar das limitações ora apresentadas, confiase que os objetivos deste estudo foram alcançados, de acordo com a Análise de Componentes Principais e com a Análise Fatorial Confirmatória, as três dimensões (status, moral e competência) corroboram com o estudo original (Lee, Whitehead, \& Balchin, 2013) e com estudos em diferentes países (Whitehead
\& Gonçalves, 2013), mostrando-se uma medida adequada para mensurar valores no esporte com a finalidade de pesquisa, mas novos estudos de validade são necessários. Os três fatores apresentaram alfas $(\alpha)$ variando de 0,55 a 0,70 no Estudo 1 e de 0,62 a 0,73 no Estudo 2. Embora alguns desses coeficientes não possam ser tidos como ideais (Nunnally, 1991; Hair et al. 2014), podem ser considerados adequados para fins de pesquisa, sobretudo quando se considera construtos psicológicos (Gouveia, Santos, \& Milfont, 2009).

Diante do exposto, é possível indicar que uma estrutura multifatorial (três fatores) da YSVQ-2 para o contexto brasileiro foi razoavelmente corroborada mediante modelagem por equações estruturais, com índices de ajuste que, embora não sejam excepcionais, podem ser considerados favoráveis, sobretudo em uma área temática e em um contexto em que são escassas as evidências sobre a estrutura fatorial de medidas desta natureza para o contexto esportivo (Hair et al. 2009).

Finalmente, sugere-se em estudos futuros que esta medida possa ser avaliada considerando amostras de atletas de alto rendimento, de diferentes modalidades esportivas e de diferentes estados brasileiros e, ainda, que novos estudos possam testar modelos alternativos (unifatorial ou bifatorial) da YSVQ-2. Além disto, pesquisas envolvendo a verificação de outros tipos de validade como a convergente-discriminante, também seriam recomendados. De modo geral, o presente estudo representa uma contribuição para a mensuração dos valores no esporte, temática carente de estudos nacionais. Esta medida pode, por exemplo, ser útil para compreender o papel dos valores dos atletas nas suas atitudes de fair play (espírito esportivo ou jogo limpo/ justo) e na adoção de comportamentos pró e antissociais no esporte. Em termos de aplicabilidade prática, essa medida pode ainda ser utilizada por psicólogos que queiram viabilizar estratégias de intervenção que priorizem ofair play e minimizem condutas agressivas e ilícitas no esporte competitivo.

\section{Referências}

Bardi, A. \& S. H. Schwartz (1994, July). Values and Behavior in Sports: A Theoretical Framework. Paper presented in $23^{\text {rd }}$ International Congress of Applied Psychology. Madri, Spain.

Boardley, I. D. \& Kavussanu, M. (2010). Effects of Goal Orientation and Perceived Value of Toughness on Antisocial Behavior in Soccer: The Mediating Role of Moral Disengagement, Journal of Sport \& Exercise Psychology, 32, 176-192. https://doi.org/10.1123/jsep.32.2.176

Braithwaite, V. A. \& Law, H. G. (1985). Structure of human values: Testing the adequacy of the Rokeach Value Survey. Journal of Personality and Social Psychology, 49, 250-263. https://doi.org/10.1037/0022-3514.49.1.250

Byrne, B. M. (2016). Structural equation modeling with Amos: basic concepts, applications and programming (3 $3^{\text {th }}$ ed.). New York: Routledge. 
Chan, Y., Whitehead, J., Hatzigeorgiadis A., \& Chow, B. (2013, July). Sport values and ethical attitudes in young Hong Kong golfers. Paper presented at the $13^{\text {th }}$ World Congress of Sport Psychology. Beijing, China.

Dodge, A. \& Robertson, B. (2004). Justifications for Unethical Behaviour in Sport: The Role of the Coach. Canadian Journal for Women Coaching Online, 4(4), 1-17.

Gonçalves, C. E., Cardoso, L., Freitas, F., Lourenço, J., \& Coelho e Silva, M. (2005) Valores no desporto de jovens: concepções, instrumentos e limitações. Boletim da Sociedade Portuguesa de Educação Física, 30(1), 93-110.

Gonçalves, M. P., Oliveira, L. C., Ribeiro, J. C. M, Barbosa, A. M. S., \& Silva, K. B. (2013, Outubro). A relação entre valores e atitudes morais no esporte. In 43ä Reunião Anual da Sociedade Brasileira de Psicologia. Aracaju, Sergipe, Brasil.

Gouveia, V. V. (1998). La naturaleza de los valores descriptores del individualismo y del colectivismo: Una comparación intra e intercultural. (Doctoral dissertation). Universidade Complutense de Madri, Espanha.

Gouveia, V. V. (2013). Teoria Funcionalista dos Valores Humanos: Fundamentos, Aplicações e Perspectivas. São Paulo: Casa do Psicólogo.

Gouveia, V. V., Santos, W. S., \& Milfont, T. L. (2009). O uso da estatística na avaliação psicológica: comentários e considerações práticas. In C. S. Hutz. (Ed.). Avanços e polêmicas em avaliação psicológica: em homenagem a Jurema Alcides Cunha (pp. 127-155). São Paulo: Casa do Psicólogo.

Hair, J. F. Jr., Black, W. C., Babin, B. J., Anderson, R. E., \& Tatham, R. L. (2009). Análise multivariada de dados (6 ${ }^{\text {th }}$ ed.). Porto Alegre, RS: Bookman.

Hodge, K. \& Lonsdale, C. (2011). Prosocial and antisocial behavior in sport: the role of coaching style, autonomous vs. controlled motivation, and moral disengagement. Journal of Sport \& Exercise Psychology, 33(4), 527-547. https://doi.org/10.1123/jsep.33.4.527

Kavussanu, M., Boardley, I. D., Sagar, S. S., \& Ring, C. (2013). Bracketed morality revisited: How do athletes behave in two contexts. Journal of Sport \& Exercise Psychology, 35(5), 449-463. https://doi.org/10.1123/jsep.35.5.449

Lee, M. J. (1996). Young people, sport and ethics: an examination of fair play in youth sport. Technical report to the Research Unit of the Sports Council. Londres.

Lee, M. J. \& Cockman, M. J. (1995). Values in children's sport: Spontaneously expressed values among young athletes. International Review for the Sociology of Sport, 30, 337-52. https://doi.org/10.1123/jsep.22.4.307

Lee, M., Whitehead J., \& Balchin, N. (2000). The measurement of values in youth sport: development of the youth sport values questionnaire. Journal of Sport and Exercise Psychology, 22, 307-326. https://doi.org/10.1123/jsep.22.4.307

Lee, M., Whitehead J., \& Balchin, N. (2013). Which sport values are most important to young people? The measurement of values in youth sport: Development of the Youth Sport Values Questionnaire. In J. Whitehead, H. Telfer \& J. Lambert (Eds.). Values in Youth Sport and Physical Education. Londres: Routledge: Taylos and Francis Group.

Lee, M. J., Whitehead J, Ntoumanis, N., \& Hatzigeorgiadis, A. (2008). Relationships among values, achievement orientations and attitudes in youth sport. Journal of Sport and Exercise Psychology, 30, 588-610. https://doi. org/10.1123/jsep.30.5.588

MacLean, J. \& Hamm, S. (2008). Values and sport participation: Comparing participant groups, age, and gender. Journal of Sport Behaviour, 31(4), 352-367.

Marôco, J. (2014). Análise de equações estruturais: fundamentos teóricos, software e aplicações. (2ạ ed.). Pêro Pinheiro: Report Number.

Nunnally, J. C. (1991). Teoría psicométrica. México, DF: Trilhas.

Nyaga, L. R. K. (2011). Valued outcomes in youth sport programs in Kenya: Towards the government's Vision 2030 (Doctoral dissertation). Springfield College, MA.

Pasquali, L. (2010). Instrumentação psicológica: Fundamentos e práticas. Porto Alegre: Artmed.

Paquali, L. (2012). Análise fatorial para pesquisadores. Brasília: LabPam.

Pitts, S. L. (2015). Sport Values of Bantam, Midget and Intermediate Female Hockey Players and Their Minor Hockey Associations (Thesis Master). Faculty Of Applied Health Sciences, St. Catharines, Ontario.

Reardon, C. L. \& Creado, S. (2014). Drug abuse in athletes. Substance abuse and rehabilitation, 5, 95-105. https://doi. org/10.2147/SAR.S53784

Rokeach, M. (1973). The Nature of Human Values. Nova York: Free Press.

Saldanha, R. P. (2012). Valores e attitudes de jovens praticantes de esportes em projetos sociais: um modelo teórico explicativo (Tese de Doutorado). Universidade Federal do Rio Grande do Sul, Porto Alegre.

Saldanha, R. P., Balbinotti, M. A. A., \& Balbinotti, C. A. A. (2015). Tradução e validade de conteúdo do Youth Sport Value Questionnaire 2. Revista Brasileira de Ciências do Esporte, 37(4), 383-388. https://doi.org/10.1016/j.rbce.2015.08.010

Schwartz, S. H. (1992) Universals in the content and structure of values: theoretical advances and empirical tests in 20 countries. In M. P. Zanna (Ed.). Advances in Experimental Social Psychology (pp. 1-65). New York: Academic Press. https://doi.org/10.1016/S0065-2601(08)60281-6

Schwartz, S. H. (2001). Existen aspectos universales en la estructura y contenido de los valores humanos? In M. Ros \& V. V. Gouveia (Eds.). Psicologia social de los valores humanos: Desarrolos teóricos, metodológicos y aplicados. Madrid: Biblioteca Nueva. 
Shields, D. L. \& Bredemeier, B. J. L. (1995). Character development through physical activity. Champaign, IL: Human Kinetics.

Sukys, S. (2010). Adaptation and validation of the Prosocial and Antisocial Behavior in Sport Scale and Youth Sport Values Questionnaire into Lithuanian. Education, Physical Training, Sport, 3(78), 97-104.

Šukys, S. \& Jansoniené, A. J. (2012). Relationship between athletes' values and moral disengagement in sport, and differences across gender, level and years of involvement. Education, Physical Training, Sport, 84(1), 55-61.

Whitehead, J. \& Gonçalves, C. E. (2013). Are sport values similar in other nations? Exploring cross-cultural value systems. In J. Whitehead, H. Telfer, \& J. Lambert (Eds.), Values in Youth Sport and Physical Education (pp. 104-118). Londres: Routledge.

Whitehead, J., Lee, M., \& Hatzigeorgiadis. (2003). Goal orientations as mediators for the personal value system. Journal of Sport Sciences, 21(4), 340-365.

Whitehead, J. (2016, Setembro). The Effect of Value Profiles on Antisocial and Prosocial Attitudes and Achievement Orientations in Youth Sport. International Convention on Science, Education and Medicine in Sport - ICSEMI, Santos, São Paulo - Brazil.

Autores:

Marina Pereira Gonçalves - Doutora, Universidade Federal do Vale do São Francisco.

Jair Nunes Rocha - Mestranda, Universidade Federal do Vale do São Francisco.

Paulo Gregório Nascimento da Silva - Mestre, Universidade Federal do Vale do São Francisco.

Letícia Coelho de Oliveira - Mestre, Universidade Federal do Vale do São Francisco.

Valeschka Martins Guerra - Doutora, Universidade Federal do Vale do Espírito Santo.

Endereço para correspondência:

Marina Pereira Gonçalves

Av: José de Sáa Maniçoba, s./n. - Centro

56304917 - Petrolina, PE, Brasil

<marinapgoncalves@gmail.com>

Recebido em: 16.08 .2017

Aceito em: 30.10 .2017 